\title{
Control of the Upper Limbs for Rehabilitative Arm-Cycling via Functional Electrical Stimulation
}

\section{Max H. Cohen, Victor H. Duenas, Christian A. Cousin, and Warren E. Dixon}

College of Engineering, University of Florida

Faculty mentor: Warren E. Dixon, Department of Mechanical and Aerospace Engineering

\begin{abstract}
Functional electrical stimulation (FES) is the application of electrical current across muscle fibers to elicit muscle contractions with the goal of achieving some function outcome (e.g. walking, cycling). FEScycling has become a very popular rehabilitative strategy over the years as it has proven to yield numerous health benefits for individuals suffering from neurological conditions. In this paper the idea of FES-cycling is extended to the upper limbs, and a new arm-cycling testbed is introduced. A dynamic model for the arm-cycle-rider system is presented, and a robust sliding-mode controller is developed for the nonlinear, autonomous, state-dependent, switched system. The controller is designed with the goal of tracking a specified crank velocity by switching between muscle stimulation and an electric motor. Despite the uncertainties and nonlinearities associated with the system, global exponential tracking of the desired crank trajectory is proven with a Lyapunov-based stability analysis. Preliminary experiments are performed with an able-bodied subject to characterize the performance of the designed controller. The results of the experiment are presented to illustrate stable tracking of the designed control system.
\end{abstract}

\section{Introduction}

Neuromuscular Electrical Stimulation (NMES) is the application of electrical current across muscle groups to artificially induce muscle contractions. NMES has become a popular research topic over the years as it has been shown to be an effective technique for rehabilitating individuals with dysfunctional muscles who have suffered from strokes, spinal cord injuries (SCI), or other neurological conditions (NC). In rehabilitative settings NMES has been used to help people with paralysis complete functional tasks such as cycling, reaching, and walking where it is referred to as functional electrical stimulation (FES). Some of the benefits of rehabilitation via FES include the recovery of muscle function, increased cardiovascular health, increased muscle mass and bone density, and increased psychological health [1]. With various health benefits, FES has been used in clinical practice for decades where stimulation is typically applied to patients in an open-loop fashion. While open-loop stimulation of muscles can yield 
some benefits associated with FES, the desire for improvement in FES performance has motivated researchers to view FES rehabilitation from a controls system perspective.

FES induced cycling has been a very popular form of FES-rehabilitation for decades now as it has shown to provide numerous physiological and psychological benefits for individuals suffering from paralysis and other ailments. Early literature on FES-cycling focused on openloop or linear proportional-derivative feedback control with the goal of tracking a desired cadence [2]. More recently, Lyapunov-based nonlinear control techniques have been developed for FES-cycling for individuals with dysfunctional limbs in their lower extremities [3]. Previous work in FES-cycling utilize a switched control input that stimulates various muscle groups at different points throughout the crank cycle according to a stimulation pattern [4]. More recently, electric motors have been added to the cycle systems to increase the controllability throughout the crank cycle and compensate for muscle fatigue [5].

Although FES of the lower limbs for cycling has been an effective rehabilitative technique for decades, motivation exists to extend the idea of FES-cycling to the upper extremities. For thousands of individuals with NCs restoring muscle function for tasks such as reaching or grabbing is essential to their well-being. Commercial FES arm-cycling devices have been developed in recent years; however, the application of closed-loop control to these systems is still an open problem. Preliminary work has been performed to characterize the dynamics of an arm-cycle system and define stimulation regions of the arms for cycling [6] which motivates the potential for closed-loop control of such a system. Although the motivation exists, there are many challenges associated with controlling FES systems.

One challenge associated with FES control is the rapid fatigue of muscles when stimulation is applied. The applied stimulation only recruits small sections of muscle fibers which cause the muscles to fatigue very quickly making the muscles difficult to control. Another challenge presented by closed-loop control of muscles via FES is the highly uncertain nature of muscles being stimulated. A few issues associated with this include the inconsistency between muscles of different individuals and unknown architecture and temperature of muscles. The results of stimulation are also affected by electrode placement, body fat, muscle fatigue, body hydration, and the unknown relation between the stimulation applied to a muscle and the force that the muscle generates. Many FES control systems are inherently switched control systems as stimulation is applied to different muscle groups at different times to coordinate functional 
motion. Switched systems present additional control challenges as switching between stable subsystems can create instabilities in the overall system [7]. All these unknown and inconsistent factors make controlling muscles via FES a challenging problem. Despite all the uncertainties, Lyapunov-based nonlinear control theory can be utilized to develop closed-loop controllers for uncertain nonlinear systems. Building on the promising results from previous FES-cycling literature as well as restorative techniques for the upper extremities, a switched-systems sliding mode controller is designed for a FES arm-cycle system with electric motor assistance with the goal of tracking a specified cadence (crank velocity).

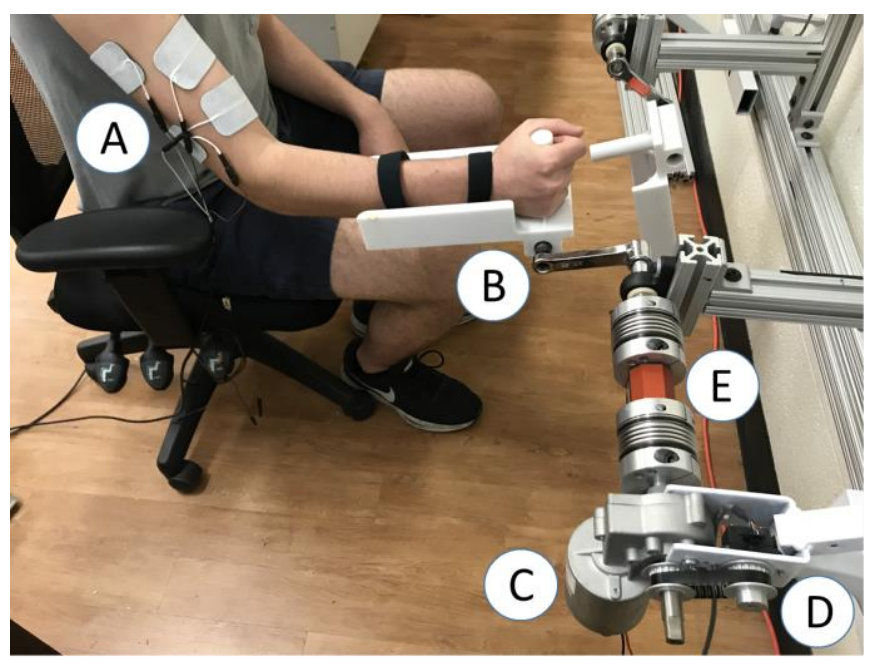

Figure 1. Experimental setup of arm-cycle test bed. A) PALS electrodes, B) crank/handle, C) DC motor, D) encoder, E) torque meter

To evaluate the performance of the designed control system, preliminary experiments are performed with one able-bodied participant. Although FES-based rehabilitation is aimed at individuals with NCs, the purpose of this experiment is to characterize the performance of the subsequently designed controller. For patient safety, it is essential that new FES control strategies and testbeds, such as the arm-cycle discussed in this paper, are first tested on ablebodied participants before conducting trials on individuals with NCs. In this paper, the goal of the experiment is for the participant to cycle at a specified crank velocity (i.e., RPM). The participant was instructed to provide no volition, and any arm movement is assumed to be a result of muscle contractions elicted using the developed controller via electrical stimulation or by activating the electric motor. The performance of the experiment will be quantified by the 
cadence tracking error. Based on the subsequently designed controller and Lyapunov stability analysis, it is hypothesized that the participant will exhibit stable tracking of the desired cadence.

\section{Arm-Cycle Dynamic Model}

The motorized arm-cycle testbed, illustrated in Fig. 1, consists of two independently controlled arm-cycles each fit with a handle, shaft, DC motor, encoder, and torque meter.

\section{Dynamics of cycle-rider system with FES}

The arm-cycle system with motor assistance can be modeled based on [8] as a single degreeof-freedom system with muscle and motor inputs as

$$
M(q) \ddot{q}+V_{m}(q, \dot{q}) \dot{q}+G(q)+P(q, \dot{q})+c_{d} \dot{q}+d(t)=\tau_{m}(q, \dot{q}, t)+\tau_{e}(q, \dot{q}, t) .
$$

In (1) $q, \dot{q}$ denotes the crank angle and velocity of the cycle, $M$ represents the inertia matrix, $V_{m}$ represents the centripetal and Coriolis effects, and $G$ represents gravitational effects. The terms $P$ and $c_{d} \dot{q}$ account for viscoelastic tissue forces and viscous damping effects, and the $d$ term denotes a time-varying disturbance. The torque applied by the stimulated muscles is denoted by $\tau_{m}(q, \dot{q}, t)$ and can be described as

$$
\tau_{m}(q, \dot{q}, t)=\sum_{m \in \mathcal{M}} B_{m} u_{m} .
$$

The term $B_{m}$ in (2) represents the uncertain, nonlinear control effectiveness term which relates the stimulation input to torque output of a muscle group where the subscript denotes each muscle group as $m \in \mathcal{M} \triangleq\{R B i, R T r i, L B i, L T r i\} . R$ and $L$ correspond to the right and left muscles, respectively, and $B i$ and Tri correspond to the biceps brachii and triceps brachii muscle groups. The intensity of input stimulation applied to each muscle group is represented by $u_{m}$. The torque applied to the system by the electric motor is denoted as $\tau_{e}$ and is described as

$$
\tau_{e}(t)=B_{e} u_{e}
$$

where $B_{e}$ is a constant that relates the motor's applied current to its resultant torque and $u_{e}$ is the controlled current applied to the motor. It is assumed that the motor constant $B_{e}$ can be bounded as $0<c_{e} \leq c_{e}$ where $c_{e}$ is a known positive constant. The viscoelastic tissue force $P(q, \dot{q})$ is a function of the torque transfer ratio of the elbow $T_{\text {elbow }}$ which can be used to analytically define stimulation regions. 


\section{Switched Systems Approach}

In many FES applications there exists a need to switch stimulation between various muscle groups throughout the activity to coordinate functional motion. For example, in the application of arm-cycles stimulation must be switched between the biceps and triceps to facilitate a cycling motion. Switching between various subsystems presents additional challenges in FES control as it can be difficult to prove the stability of an overall switched system, despite having stable subsystems. In early FES-cycling literature, stimulation is applied to each muscle group in regions of the crank cycle where the muscles can contribute to forward motion, and stimulation is not applied in regions where the muscles do not contribute much to forward movement [3]. The regions of the crank cycle where stimulation is not applied are referred to as the uncontrolled regions or kinematic dead zones, and in more recent literature electric motors have been coupled to cycling testbeds to facilitate the controllability of the kinematic dead zones [5]. To maximize rehabilitation, it is preferred that the user exerts much of the effort needed to complete the cycling task (via muscle stimulation), so the motor is only activated in the defined kinematic dead zones. Switching stimulation between various muscle groups as well as switching the control input from stimulator to motor makes the overall arm-cycle system an autonomous, statedependent, switched control system.

In the following switched systems analysis, let $\mathbb{Q}$ be the set of crank angles contained between $[0,2 \pi)$, let $Q_{m} \subset \mathbb{Q}$ represent the regions of the crank cycle where stimulation is applied, and let $Q_{e} \subset \mathbb{Q}$ represent the regions of the crank cycle where the electric motor takes over. For the armcycle system $Q_{m}$ can be defined for the biceps and triceps muscles as regions in which $T_{\text {elbow }}$ is above some threshold indicating kinematically efficient forward pedaling. Fig. 2 illustrates an example schematic of the stimulation regions. The motor controlled regions can be defined as

$$
\begin{aligned}
& Q_{F E S} \triangleq \bigcup_{m \in \mathcal{M}} Q_{m} \\
& Q_{e} \triangleq \mathbb{Q} / Q_{F E S} .
\end{aligned}
$$




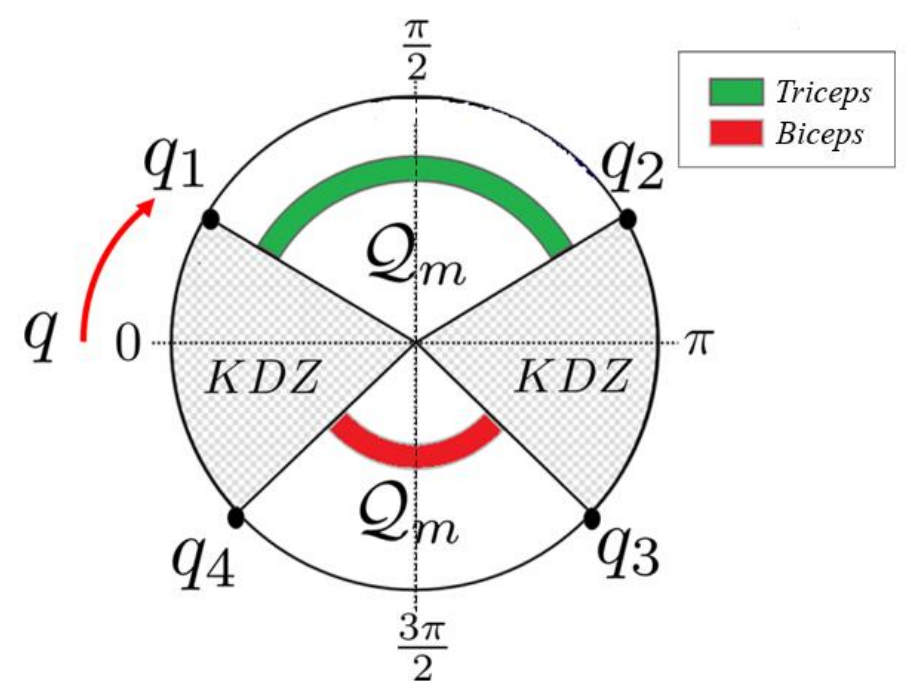

Figure 2. Example schematic of the crank angles where the upper-limb muscles are activated (colored bands). The gray areas, labeled as kinematic dead zones (KDZ), denote the crank angles where muscles are not activated, but where the electric motor is to facilitate overall controllability.

Based on the defined switching laws, a switching signal can be developed for the muscle groups $\sigma_{m}$ and motor $\sigma_{e}$ as

$$
\begin{gathered}
\sigma_{m} \triangleq \begin{cases}1, & \text { if } q \in Q_{m} \\
0, & \text { if } q \notin Q_{m}\end{cases} \\
\sigma_{e} \triangleq \begin{cases}1, & \text { if } q \in Q_{e} \\
0, & \text { if } q \notin Q_{e}\end{cases}
\end{gathered}
$$

Using these state-dependent switching signals defined in (6), control inputs for both muscle stimulation and current input to the electric motor are defined as

$$
\begin{gathered}
u_{m} \triangleq k_{m} \sigma_{m} u, \\
u_{e} \triangleq k_{e} \sigma_{e} u,
\end{gathered}
$$

where $k_{m}, k_{e}$ are positive, constant control gains and $\mathrm{u}$ is the control input, which will be designed in the following section. The dynamics of the overall system can be rewritten, taking into account the switching signal as

$$
M \ddot{q}+V_{m} \dot{q}+G+P+c_{d} \dot{q}+d_{t}=B_{\sigma} u,
$$

where $B_{\sigma}$ denotes the lumped, switched control effectiveness defined as

$$
B_{\sigma} \triangleq \sum_{m \in \mathcal{M}} b_{m} k_{m} \sigma_{m}+b_{e} k_{e} \sigma_{e}
$$


Since four muscle groups will be stimulated ( $R B i, R T r i, L B i, L T r i)$, there are $2^{4}=32$ possible combinations of stimulated muscle groups. The overall switched system abides by the following properties:

Property 1: The inertia matrix $M$ is positive definite and can be bounded as $c_{m} \leq M \leq c_{M}$, where $c_{m}$ and $c_{M}$ denote known constants.

Property 2: The centripetal/Coriolis matrix $V_{m}$ can be upper bounded as $\left|V_{m}\right| \leq c_{V}|\dot{q}|$, where $c_{V}$ is a known constant.

Property 3: The gravitational effects matrix $G$ can be upper bounded as $|G| \leq c_{G}$, where $c_{G}$ is a known constant.

Property 4: The passive and viscoelastic tissue effects $P$ can be bounded as $|P| \leq c_{P 1}+$ $c_{P 2}|\dot{q}|$, where $c_{P 1}$ and $c_{P 2}$ are known constants.

Property 5: The time-varying disturbance term $d$ can be upper bounded as $|d| \leq c_{d}$, where $c_{d}$ is a known constant.

Property 6: The lumped, switched control effectiveness term $B_{\sigma}$ can be bounded as $c_{B 1} \leq$ $B_{\sigma} \leq c_{B 2}, \forall \sigma$, where $c_{B 1}$ and $c_{B 2}$ are known constants.

Property 7: The inertia and centripetal/Coriolis matrices follow the skew-symmetry relationship such that $\frac{1}{2} \dot{M}-V_{m}=0$.

\section{Control Development}

The primary control objective is for the rider to cycle at a desired crank cadence with performance quantified by the following error signals

$$
\begin{gathered}
e_{1} \triangleq q_{d}-q, \\
e_{2} \triangleq \dot{e}_{1}+\alpha e_{1},
\end{gathered}
$$

where $e_{1}$ represents the position tracking error, and $e_{2}$ is a filtered tracking error considering errors in position and cadence tracking. The term $q_{d}$ is the desired crank position which is designed such that its derivatives exist and are bounded as $\dot{q}_{d}, \ddot{q}_{d} \in \mathcal{L}_{\infty}$, and $\alpha$ is a user-defined positive gain. The dynamics of the arm-cycle are injected into the error system by taking the time derivative of (12), multiplying by the inertia matrix $M$, and utilizing (9), (11), and (12) to yield the open-loop error system as

$$
M \dot{e}_{2}=\chi-e_{1}-V_{m} e_{2}-B_{\sigma} u,
$$


where the term $\chi$ in (13) denotes a bucket of constant and state-dependent terms which can be bounded using a known function of the states using the above properties. For the subsequent analysis, let $z$ be an error vector defined as

$$
z \triangleq\left[e_{1} e_{2}\right]^{T}
$$

Based on the open-loop error system from (13) and the subsequent Lyapunov-based stability analysis, a robust sliding mode controller is designed as

$$
u \triangleq k_{1} e_{2}+\left(k_{2}+k_{3}\|z\|+k_{4}(\|z\|)^{2}\right) \operatorname{sgn}\left(e_{2}\right),
$$

where $\operatorname{sgn}(\cdot)$ denotes the signum function and $k_{1}, k_{2}, k_{3}, k_{4}$ are selectable positive control gains. Substituting the control input into the open-loop error system yields the closed-loop system dynamics

$$
M \dot{e}_{2}=\chi-e_{1}-V_{m} e_{2}-B_{\sigma}\left[k_{1} e_{2}+\left(k_{2}+k_{3}\|z\|+k_{4}(\|z\|)^{2}\right) \operatorname{sgn}\left(e_{2}\right)\right] .
$$

\section{Stability Analysis}

Let $V_{L}$ represent a positive-definite, continuously differentiable Lyapunov function candidate defined as

$V_{L}$ can be bounded as

$$
V_{L} \triangleq \frac{1}{2} M e_{2}^{2}+\frac{1}{2} e_{1}^{2}
$$

$$
\lambda_{1}(\|z\|)^{2} \leq V_{L} \leq \lambda_{2}(\|z\|)^{2}
$$

where $\lambda_{1}, \lambda_{2}$ are known constants. Taking the derivative of $V_{L}$ yields

$$
\dot{V}_{L}=e_{2}\left(\chi-e_{1}-V_{m} e_{2}-B_{\sigma} u\right)+\frac{1}{2} \dot{M} e_{2}^{2}+e_{1}\left(e_{2}-\alpha e_{1}\right)
$$

Utilizing Property 7, canceling terms, substituting (15), utilizing Property 6, and upper bounding yields

$$
\dot{V}_{L} \leq-c_{B 1} k_{1} e_{2}^{2}-\alpha e_{1}^{2}
$$

provided certain gain conditions are met. $\dot{V}_{L}$ can then be expressed in terms of $z$ as

$$
\dot{V}_{L} \leq-\lambda_{3}(\|z\|)^{2}
$$

where $\lambda_{3}$ is a known constant. Recognizing how $V_{L}$ is bounded in (18) allows for $\dot{V}_{L}$ to be expressed as a first order differential equation of the form

$$
\dot{V}_{L} \leq \frac{\lambda_{3}}{\lambda_{2}} V_{L}
$$


Solving (22) yields

$$
V_{L} \leq V_{L, 0} \exp \left(\frac{-\lambda_{3}}{2 \lambda_{2}} t\right)
$$

where $V_{L, 0}$ is the initial condition of $V_{L}$. Recognizing the bounding conditions on $V_{L}$ and rearranging terms allows (23) to be expressed as

$$
\|z\| \leq \sqrt{\frac{\lambda_{2}}{\lambda_{1}}}\left\|z_{0}\right\| \exp \left(\frac{-\lambda_{3}}{2 \lambda_{2}} t\right),
$$

where $z_{0}$ is the initial condition of the error vector. Based on the stability analysis, the closedloop error system in (16) is globally, exponentially stable for all $t \in\left[t_{o}, \infty\right)$ where $t_{0}$ denotes the initial time. This global exponential result is illustrated by (24) where $z$ exponentially converges to 0 as time goes to infinity.

\section{Experiments}

Preliminary experiments were performed to evaluate the performance of the controller in (15). In these experiments only the right biceps and triceps muscle groups are stimulated with the goal of tracking a desired crank velocity of 65 RPM. The experiments were performed with an ablebodied subject (age 21). Prior to participation, written informed consent was obtained from the participant, as approved by the Institutional Review Board at the University of Florida.

\section{Testbed Setup}

The arm-cycle testbed was specifically designed for FES cycling experiments. A $60 \mathrm{~W}, 100 \mathrm{~V}$, DC motor is fixed to the frame and attached to the crank shaft of the testbed. An encoder (US Digital H1) provides position feedback of the crank. The user is strapped into the handle to ensure he/she is fixed to the crank. Current to the motor is controlled with an Advanced Motion Controls motor driver in conjunction with data acquisition hardware (Quanser QPID). The controller is implemented using real-time software (MATLAB/Simulink, QUARC 2.5) on a desktop computer running Windows 10 with a sampling rate of $500 \mathrm{~Hz}$. Stimulation was delivered to the participant via a Hasomed RehaStim stimulator and PALS electrodes (1.5" $\mathrm{x}$ 3.5") placed on the biceps and triceps muscle groups. The stimulation was controlled with the desktop computer and real-time software. Fig. 1 depicts the testbed and experimental setup. The 
testbed is also fit with an emergency stop button allowing the user to stop the experiment at any point.

\section{Experimental Setup}

Electrodes were placed on the participant's right biceps and triceps muscle groups, and the participant was seated in a chair in front of the testbed. The participant was placed close enough so that a crank cycle could be completed without having to fully extend the arm. The participant was instructed to stay fixed in the chair and to remain completely passive so that there was no volitional effort.

The objective of the experiment was for the controller to track a crank velocity of 65 RPM by switching between muscles and motor to coordinate forward motion. The desired trajectory function was designed to exponentially increase to 65 RPM and then level out. The software was designed to have the motor bring the cycle up to speed, then have the switching begin once the velocity reached its desired value. For each trial the switching signal was designed to activate at approximately 40 seconds. After the 40 second mark the participant receives electrical stimulation for the remainder of the trial. The trial is designed to be 180 seconds long. For this experiment the stimulation regions were defined experimentally and can be expressed as

$$
\begin{gathered}
290^{\circ}<Q_{b}<330^{\circ} \\
95^{\circ}<Q_{t}<130^{\circ} .
\end{gathered}
$$

where $Q_{b}$ and $Q_{t}$ denote the regions in which the biceps and triceps are stimulated, respectively. The motor was given a current offset of 0.05 A so that it was not completely shut off in the muscle regions to smooth out the cycling. Multiple trials were run to tune the gains in (12) and (15) such that the controller could adequately track the desired cadence. With the gains tuned one final trial was run and data was collected, the results of which are displayed in Fig. 3 and Fig. 4. 


\section{Results}
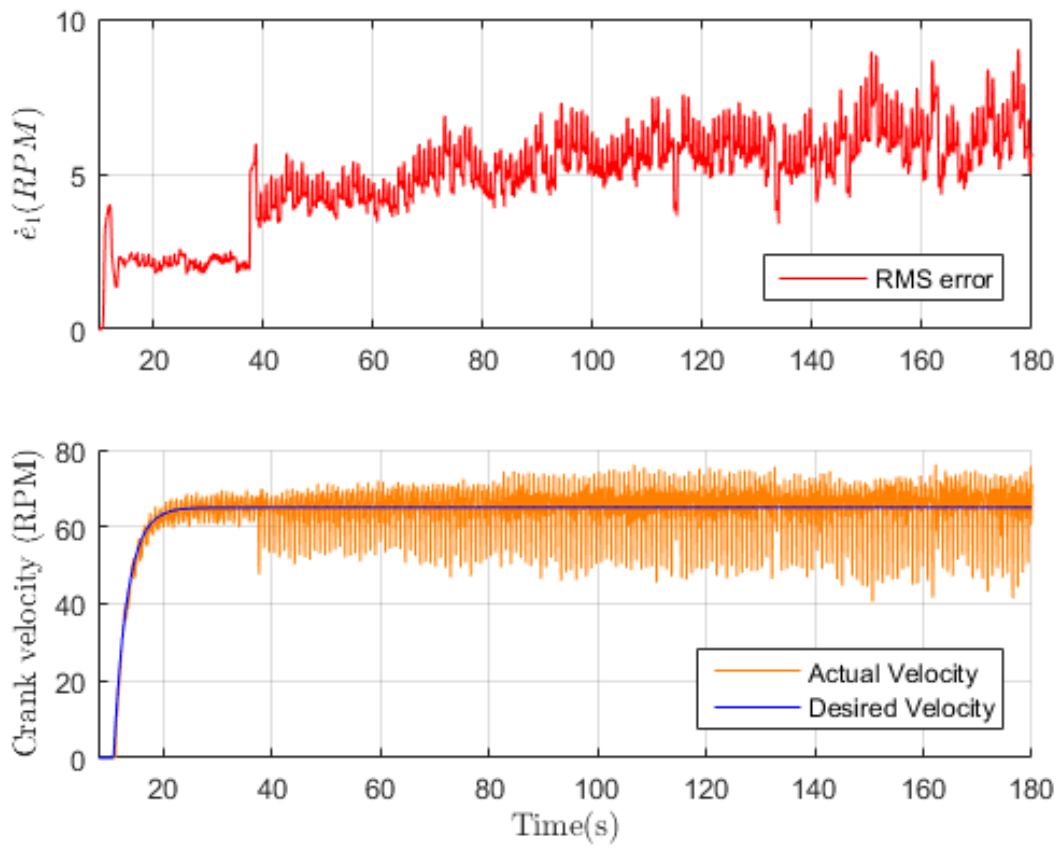

Figure 3. Tracking performance quantified by the root mean square (RMS) of the cadence tracking error (top). RMS stems from the difference between the desired and actual velocity (bottom). Stimulation begins at approximately 40 seconds. Error increases with time due to fatigue of the muscles being stimulated.
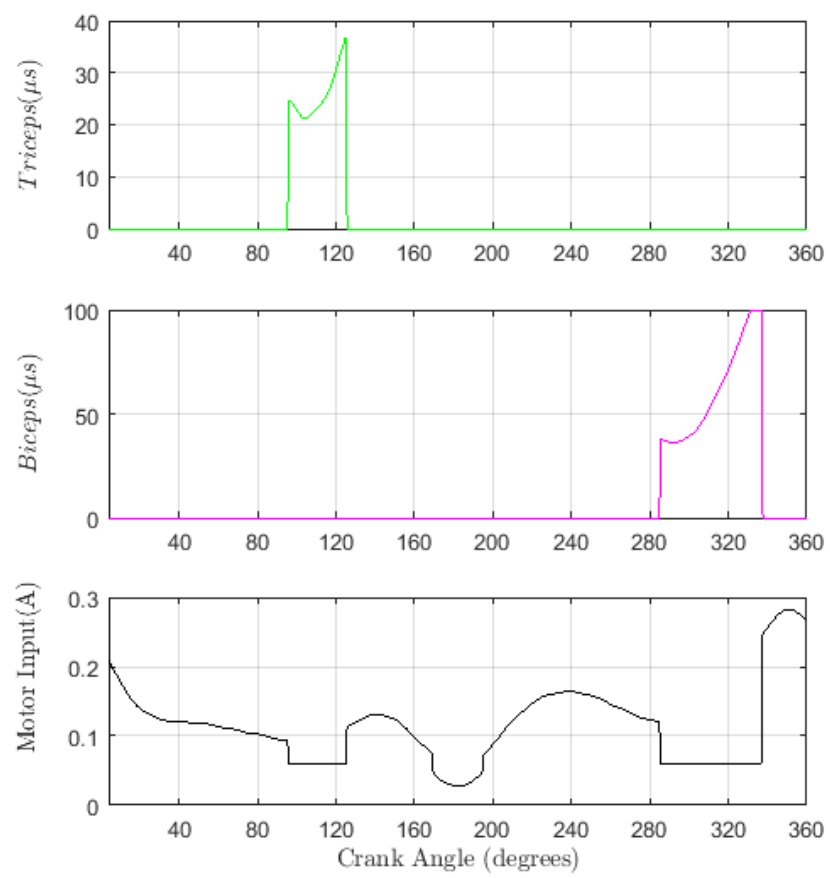

Figure 4. FES stimulation intensities and motor input for one crank cycle (0-360 degrees). Stimulation is delivered to the triceps (green) and biceps (purple) in the muscle regions of the crank cycle and the motor is activated in the kinematic dead zones. 
The performance of the controller is characterized by the tracking performance, illustrated in Fig. 3. It can be seen that in first 40 seconds of the experiment (when only the motor is activated) the control system exhibits a tracking error of approximately \pm 5 RPM and a root mean square (RMS) error of approximately 2.5 RPM. When the stimulation is turned on at the 40 second mark, the error increases as indicated by the error envelope in Fig. 3 (bottom) and the jump in RMS error to approximately 5 RPM. The error then slowly increases for the remainder of the experiment to about 7.5 RPM. Fig. 4 presents the stimulation intensities for each muscle group and motor input over the period of one crank cycle.

\section{Discussion}

The results of the experiments illustrated in Fig. 3 and Fig. 4 indicate stable tracking of a desired cycling cadence. The controller predicted that the tracking error would exponentially converge to zero; however, in reality the error exponentially converges to some ultimate bound. This discrepancy is likely due to unmodeled effects of the muscles and the lack of compensation for input delay of the muscle. It is important to note that tracking performance decreases over the course of the experiment as the muscles become fatigued. It is also important to note that the overall system remains stable despite switching between multiple subsystems.

\section{Conclusion}

The benefits and challenges associated with controlling FES systems are introduced, and a new FES arm-cycling testbed is presented. A dynamic model is developed for an FES arm-cyclerider system. Based on this dynamic model a robust sliding-mode controller is designed with the goal of tracking a specified cadence. Preliminary experiments are performed with an able-bodied subject to characterize the performance of the controller. The results indicate the controller can sufficiently track a desired cadence, despite switching between various subsystems.

This arm-cycling testbed will allow further research into FES rehabilitation of the upper limbs. The testbed and designed controller have the potential to restore muscle function and greatly increase the well-being of individuals suffering from certain neurological conditions. Future work will focus on improving performance by analytically defining stimulation regions through a kinematic analysis of the upper arms and implementing adaptive control schemes. Moving forward, experiments will involve independent control of both arms for individuals with neurological conditions. 


\section{References}

[1] C.-W. Peng, S.-C. Chen, C.-H. Lai, C.-J. Chen, C.-C. Chen, J. Mizrahi, and Y. Handa, "Review: Clinical benefits of functional electrical stimulation cycling exercise for subjects with central neurological impairments," J. Med. Biol. Eng., vol. 31, pp. 1-11, 2011.

[2] D.J. Pons, C. L. Vaughan, and G. G. Jaros, "Cycling device powered by the electrically stimulated muscles of paraplegics," Med. Biol. Eng. Comput., vol. 27, no. 1, pp. 1-7, 1989.

[3] M. J. Bellman, T. -H. Cheng, R. J. Downey, C. J. Hass, and W. E. Dixon, "Switched Control of Cadence During Stationary Cycling Induced by Functional Electrical Stimulation," IEEE Transactions on Neural Systems and Rehabilitation Engineering, Vol. 24, No. 12, pp. 13731383 (2016).

[4] W. E. Dixon and M. J. Bellman, "Cycling Induced by Functional Electrical Stimulation: A Control Systems Perspective," ASME Dynamic Systems \& Control Magazine, Vol. 4, No. 3, pp. 3-7 (2016).

[5] M. J. Bellman, R. J. Downey, A. Parikh and W. E. Dixon, "Automatic Control of Cycling Induced by Functional Electrical Stimulation with Electric Motor Assistance," IEEE Transactions on Automation Science and Engineering, Vol. 14, No. 2, pp. 1225-1234 (2017).

[6] D. Liberzon, Switching in Systems and Control. Birkhauser, 2003.

[7] A. Carraha, C. Cousin, V. Duenas, C. Rouse, and W. Dixon, "Stationary Arm Cycling Induced by Functional Electrical Stimulation - Muscle Zone Characterization," Journal of Undergraduate Research, University of Florida, Vol. 18, Issue 3, 2017.

[8] V. H. Duenas, C. A. Cousin, A. Parikh, P. Freeborn, E. J. Fox, and W. E. Dixon, "Motorized and Functional Electrical Stimulation Induced Cycling via Switched Repetitive Learning Control," IEEE Transactions on Control Systems Technology, to appear. 